\title{
Development of an activity disease score in patients with uveitis (UVEDAI)
}

\author{
Esperanza Pato ${ }^{1} \cdot \mathrm{M}^{\mathrm{a}}$ Auxiliadora Martin-Martinez ${ }^{2} \cdot$ Adela Castelló $^{3} \cdot$ Rosalía Méndez-Fernandez $^{4,5} \cdot$ \\ Santiago Muñoz-Fernández ${ }^{6}$ Miguel Cordero-Coma ${ }^{7} \cdot$ Lucia Martinez-Costa $^{8} \cdot$ Elia Valls $^{9} \cdot$ Miguel Reyes $^{10}$. \\ Félix Francisco $^{11}$ • Mar Esteban ${ }^{12}$ - Alex Fonollosa ${ }^{13}$ - Fernando Sanchez-Alonso ${ }^{2}$ - Cruz Fernández-Espartero ${ }^{14}$. \\ Teresa Diaz-Valle $^{15}$ • José Miguel Carrasco ${ }^{16}$ • Emma Beltran-Catalán ${ }^{17}$ • Marisa Hernández-Garfella ${ }^{18}$. \\ María Victoria Hernández $^{19}$ - Laura Pelegrin ${ }^{20}$ - Ricardo Blanco ${ }^{21} \cdot$ David Diaz-Valle $^{4,5}$
}

Received: 18 May 2016 / Accepted: 22 October 2016 / Published online: 4 November 2016

(c) The Author(s) 2016. This article is published with open access at Springerlink.com

\begin{abstract}
To develop a disease activity index for patients with uveitis (UVEDAI) encompassing the relevant domains of disease activity considered important among experts in this field. The steps for designing UVEDAI were: (a) Defining the construct and establishing the domains through a formal judgment of experts, (b) A two-round Delphi study with a panel of 15 experts to determine the relevant items, (c) Selection of items: A logistic regression model was developed that set ocular inflammatory activity as the dependent variable. The construct "uveitis inflammatory activity" was defined as any intraocular inflammation that
\end{abstract}

Electronic supplementary material The online version of this article (doi:10.1007/s00296-016-3593-1) contains supplementary material, which is available to authorized users.

Esperanza Pato

esperanza.pato@salud.madrid.org

1 Rheumatology Department and Health Research Institute (IdISSC), Hospital Clínico San Carlos, C/ Profesor Martin Lagos s/n, 28040 Madrid, Spain

2 Research Unit of Spanish Society of Rheumatology, Madrid, Spain

3 Cancer Epidemiology Unit, National Center for Epidemiology, Instituto de Salud Carlos III, Madrid, Spain

4 Ophthalmology Department and Health Research Institute (IdISSC), Hospital Clínico San Carlos, Madrid, Spain

5 Cooperative Research Network on Age-Related Ocular Pathology, Visual and Life Quality, Instituto de Salud Carlos III, Madrid, Spain

6 Rheumatology Department, Hospital Universitario Infanta Sofia, Universidad Europea de Madrid, San Sebastian de los Reyes, Madrid, Spain

7 Ophthalmolgy Department, Hospital Universitario de León, León, Spain included external structures (cornea) in addition to uvea. Seven domains and 15 items were identified: best-corrected visual acuity, inflammation of the anterior chamber (anterior chamber cells, hypopyon, the presence of fibrin, active posterior keratic precipitates and iris nodules), intraocular pressure, inflammation of the vitreous cavity (vitreous haze, snowballs and snowbanks), central macular edema, inflammation of the posterior pole (the presence and number of choroidal/retinal lesions, vascular inflammation and papillitis), and global assessment from both (patient and physician). From all the variables studied in the multivariate model, anterior chamber cell grade, vitreous haze, central macular edema, inflammatory vessel sheathing, papillitis, choroidal/retinal lesions and patient evaluation were

8 Ophthalmolgy Department, Hospital Universitario Doctor Peset, Valencia, Spain

9 Rheumatology Department, Hospital Universitario Doctor Peset, Valencia, Spain

10 Ophthalmolgy Department, Hospital de Gran Canaria Doctor Negrin, Gran Canaria, Spain

11 Rheumatology Department, Hospital de Gran Canaria Doctor Negrin, Gran Canaria, Spain

12 Ophthalmology Department, Hospital Universitario Infanta Sofia, San Sebastian de los Reyes, Madrid, Spain

13 Ophthalmology Department, BioCruces Health Research Institute, Cruces University Hospital, University of the Basque Country, Barakaldo, Spain

14 Rheumatoloy Department, Hospital Universitario de Móstoles, Móstoles, Madrid, Spain

15 Ophthalmology Department, Hospital Universitario de Móstoles, Móstoles, Madrid, Spain

16 ATLANTES Research Programme, Institute for Culture and Society, University of Navarra, Pamplona, Spain 
included in UVEDAI. UVEDAI is an index designed to assess the global ocular inflammatory activity in patients with uveitis. It might prove worthwhile to motorize the activity of this extraarticular manifestation of some rheumatic diseases.

Keywords Uveitis - Activity index - Ocular inflammation · Outcome measures

\section{Introduction}

Uveitis is defined as inflammation of the uvea, the vascular middle layer of the eye. The term refers to a great number of diseases characterized by intraocular inflammation involving the uvea and other ocular structures (retina, vitreous cavity and retinal vessels) [1]. It may be characterized as having a poor visual prognosis and remains one of the leading blinding disorders [2,3]. Uveitis is an extraarticular manifestation frequently found in many rheumatic diseases (spondyloarthropathy, Behçet, sarcoidosis, lupus, vasculitis, etc.) and may cause significant morbidity. In some patients, such as those with Behçet disease or spondyloarthritis, uveitis is often the origin of the main symptoms of the disease. Consequently, the implementation of interdisciplinary units-ophthalmology and rheumatology-assumes considerable relevance in the assessment and management of these patients.

The Standardization of Uveitis Nomenclature Working Group [4, 5] (SUN) developed criteria based on the primary anatomic location of inflammation within the eye (International Uveitis Study Group [6]), as well as criteria for onset, duration, course and disease activity. The SUN criteria for the classification of uveitis have become the standard when publishing uveitis data, ensuring greater analytical rigor when comparing studies. However, such criteria only take into account inflammation in the anterior chamber and vitreous haze when defining uveitis activity.

Standardized and validated outcome measures of disease activity are lacking in uveitis management, which makes it difficult to compare efficacy and response to treatment

17 Rheumatoloy Department, Hospital General Universitario de Valencia, Valencia, Spain

18 Ophthalmology Department, Hospital General Universitario de Valencia, Valencia, Spain

19 Rheumatology Department, Hospital Clínic of Barcelona, Barcelona, Spain

20 Ophthalmology Department, Hospital Clínic of Barcelona, Barcelona, Spain

21 Rheumatology Department, Hospital Universitario de Valdecilla, Santander, Spain
[7-9]. In addition, the activity of uveitis is not recorded by the usual articular indexes that are used by rheumatologists to monitoring disease activity and function such as BASDAI, BASFI or ASDAS. In this line, Denniston et al. [9] carried out a systematic review of clinical trial or treatments for uveitis, highlighting the heterogeneity of primary outcomes and arguing that the complex issue of selecting outcome measures for clinical trials of efficacy related to uveitis needs to be addressed.

As in rheumatology, the validation of outcome measures and the development of a disease activity index [10-12] can be a major advance in clinical trials as well as in evaluation of treatment response of patients with uveitis in daily clinical practice.

The objectives of the current study were to develop a disease activity index for patients with uveitis (UVEDAI) that would include relevant domains of disease activity considered important among experts in uveitis.

\section{Methods}

\section{Development of UVEDAI}

The index has been developed according to the criteria described in the Outcome Measures in Rheumatology Clinical Trials (OMERACT) [13].

A formal interdisciplinary working group for uveitis, consisting of eight ophthalmologists and seven rheumatologists, was formed. The participants had relevant experience in the management of all types of uveitis and work in interdisciplinary uveitis units at hospitals in the Spanish national health system. Chronological steps to design UVEDAI:

1. Definition of the construct and establishment of domains: The consensus technique used was the formal judgment of experts, which represents the informed opinion of people with experience in the subject, who are recognized by others as skilled in the topic, and who can provide information, evidence, judgments and assessments [14]. A consensus meeting took place in October 2011 in Madrid, coordinated by a methodologist with training and experienced in this technique, and all the members participated. First, one or two indices of activity, based on the anatomic location, were discussed, which the expert panel decided by consensus to build into a global index. Second, the construct "uveitis inflammatory activity" was defined as any intraocular inflammation including external structures (cornea) in addition to uvea, and the domains and items were established. Seven domains were identified: bestcorrected visual acuity (BCVA), inflammation of the 
anterior chamber, intraocular pressure, inflammation of the vitreous cavity, central macular edema (ME), inflammation of the posterior pole and global assessment.

2. Determination of items: The panel selected 15 relevant items that would thereafter be tested to derive the new disease activity index. A two-round Delphi consensus technique was used by the panel to achieve consensus. Agreement on the utility of each item to assess "uveitis inflammatory activity" was evaluated using a Likert scale (1 [strongly disagree] to 5 [strongly agree]). The degree of agreement (DA) among the working group for each item was high (DA > 85\%). In the first round, 12 items reached DA $>85 \%$ and the three remaining reached DA $>85 \%$ after the second round, and thus were included in UVEDAI.

As result, seven domains and 15 items were identified: best-corrected visual acuity, inflammation of the anterior chamber (anterior chamber (AC) cells, hypopyon, the presence of fibrin, active posterior keratic precipitates and iris nodules: five items), intraocular pressure, inflammation of the vitreous cavity (vitreous haze, snowballs and snowbanks: three items), central macular edema, inflammation of the posterior pole (the presence and number of choroidal/retinal lesions, vascular inflammation and papillitis: three items) and global assessment from both the patient and physician (two items).

3. Selection of items: A logistic regression model was constructed, the ordinal dependent variable of which was ocular inflammatory activity (with three categories: mild, moderate and severe) and as variables predictive, the items mentioned above. After building the first model, the variables were eliminated in inverse order of their significance level to obtain the minimal model explaining the maximum variability. The capability of the final model to discriminate patients with uveitis according to the level of inflammatory activity was then determined.

\section{Items and operational definitions}

Inflammatory activity of uveitis was considered the primary endpoint. This variable was defined as any intraocular inflammation, and it was categorized as mild, moderate and severe at the discretion of the ophthalmologist following patient assessment. The manner in which each hospital carried out the evaluations was homogenized, and the CIRRUS spectral domain (Carl Zeiss) was the optical coherence tomography (SD-OCT) equipment used in all centers.

Clinical evaluations included visual acuity (best-corrected Snellen visual acuity) and ophthalmic examination. A slit-lamp examination was used to evaluate the anterior segments. AC cells were graded according to the classification established by the SUN [4]. Intraocular pressure [15] was determined by using a contact tonometer (Goldmann). Indirect ophthalmoscopy was also performed in all patients to evaluate the vitreous and posterior segments [16]. The Nussenblatt scale [17] was adapted for any vitreous haze that had been graded as mild or severe $(1-2+$ or $3-4+$, respectively). SD-OCT was used in all patients to determine the presence of macular edema [18], which was defined as a central retinal thickness greater than $315 \mu \mathrm{m}$. The 1-mm central retinal thickness was evaluated using the macular cube strategy.

An overall assessment of the disease by both patient and researcher was made using a visual analog scale (VAS) of $0-10 \mathrm{~cm}, 0$ being the lowest (best) possible disease activity and 10 the highest (worst). VAS assessments of patient and physician were performed with the following questions; VAS patient: "at the present time, how would you rate your eyesight using both eyes (with glasses or contact lenses, if you wear them): excellent, good, fair, poor, or very poor, or are you completely blind". VAS physician: "taking into account eye inflammation and macular edema, indicate your assessment of disease activity experienced by this patient".

\section{Selection and recruitment of patients}

Patients diagnosed with uveitis at nine multidisciplinary uveitis units in hospitals from Spanish National Health System were invited to participate in the study. The recruitment period was from March 2013 to July 2014. Consecutive patients were eligible for inclusion during the recruitment period, with one out of two patients who met the selection criteria being enrolled until the sample size was reached. Inclusion criteria consisted of the following: signed informed consent by the patient or his/her legal representative, aged five years or older, patients diagnosed with active anterior, intermediate, posterior uveitis or panuveitis in at least one eye, and with active uveitis in at least one eye at the time of the selection visit. We excluded patients in complete uveitis remission, those who had been diagnosed with surgical or traumatic endophthalmitis, and any patients then participating in a clinical trial or research project related to this or other health problems. For those patients with bilateral uveitis, we took into account the fact that treatment decisions would always be based on the most inflamed eye. This study was performed following the principles outlined in the Helsinki Declaration, and the study protocol was approved by the ethics committee at all participating hospitals.

The sample size was calculated based on at least 10 events per variable, as suggested in the current literature [19-21]. The presence of moderate or severe uveitis was regarded as an event. When the expected prevalence of such an event 
reached $60 \%$, we determined that 200 patients would be sufficient for designing a model that would include up to 12 variables (200 patients $\times 60 \%=120$ expected events, which corresponds to $120 / 10$ possible variables).

\section{Statistical analysis}

The categorical variables were described using frequencies and percentages. These were compared among activity levels using Chi-squared tests, and Fisher's exact tests otherwise. The variables that followed a normal distribution were described using the mean and standard deviation (SD), and the differences between groups of activity were assessed using ANOVA tests. Non-normally distributed variables were described with the median and interquartile interval (IQR), and differences between groups were assessed with the Kruskal-Wallis nonparametric test. In all the analyses, a $p$ value $<0.05$ was considered statistically significant.

Given the ordinal nature of the outcome variable (uveitis: mild, moderate and severe), we adjusted the model using ordinal logistic regression. We followed the stepwise methodology, including the potentially explanatory variables in the model, by statistical significance order. The selection of independent variables in the multivariate models was based on clinical judgments and those with a $p$ value $<0.20$ in the bivariate analysis. After building the first model, we tested the deletion of each variable in inverse significance order to obtain the minimum model that would explain the maximum variability. The inclusion or exclusion of each variable was determined using a likelihood ratio test (LRT) with a 90\% confidence level. The assumption of proportional odds across responses underlying the ordered logistic models was also verified using the approximate LRT with the same confidence level. Confidence intervals for the odds ratio were calculated at a $95 \%$ confidence level.

Regression coefficients in logarithmic format for the multivariate model were used to calculate the score used to define the ocular activity level of a given patient:

$$
\begin{gathered}
\text { UVEDAI }=\sum_{i=1}^{v} \beta_{i} \cdot x_{i} \\
v=\text { Number of variables included } \\
\beta_{i}=\text { Logarithmic regression coefficient for variable } i \\
\quad x_{i}=\text { Variable } i
\end{gathered}
$$

We used the resulting score to calculate a patient's probability of being classified with mild, moderate or severe uveitis based on the following cumulative probabilities:
From which we calculated probabilities for the three categories:

$$
\begin{aligned}
\operatorname{Pr}(\text { uveitis }= & \text { Mild })=\operatorname{Pr}(\text { uveitis }<\text { Moderate }) \\
\operatorname{Pr}(\text { uveitis }= & \text { Moderate })=\operatorname{Pr}(\text { uveitis }<\text { Severe }) \\
& -\operatorname{Pr}(\text { uveitis }<\text { Moderate }) \\
\operatorname{Pr}(\text { uveitis }= & \text { Severe })=1-\operatorname{Pr}(\text { uveitis }<\text { Severe })
\end{aligned}
$$

The patient was then classified in that category with the higher probability (see example in Supplementary Material).

The modeling accuracy was assessed using the area under the curve (AUC) [22-24]. To overcome any limitations in the use of AUC for binary outcomes, and basing our calculations on proportional odds, we formulated two AUC measures of uveitis: one for discriminating mild versus moderate and severe activity levels and another for mild and moderate versus severe activity. In order to provide a measurement of the uncertainty of the model's accuracy, we calculated the $95 \%$ percentile confidence intervals for the estimation of both AUCs by performing a nonparametric bootstrap estimation with 1000 replications. Using sampling replacement, the bootstrap obtained 1000 replicates of the original dataset and coefficients of the final model. The corresponding AUCs were then estimated over each of these replicates. The $95 \%$ percentile confidence intervals for each AUC were represented by percentiles 2.5 and 97.5 of the 1000 bootstrap point estimates' distribution.

Additionally, the internal validity of the results was assessed by comparing the estimation of the AUC with an empirical distribution of the outcome in order to determine the probability of obtaining a better model accuracy by chance. For that purpose, we calculated 1000 replicates of the original outcome variable containing the exact number of cases of mild $(n=77)$, moderate $(n=93)$ and severe ( $n=25)$ uveitis, but randomly distributed. The discrimination of mild versus moderate + severe diagnosis and the discrimination of mild + moderate versus severe diagnosis were assessed for each of the 1000 random replicates by calculating the AUC. The probability of obtaining by chance an AUC better than the original was calculated, dividing by 1000 the total number of cases in which the random AUC was better than the original AUC.

\section{Results}

Our initial sample contained 203 patients. After excluding missing values $(n=8)$, a total of 195 patients were

$\operatorname{Pr}($ uveitis $<$ moderate $)=\left[1 /\left(1+e^{(\text {score-constant mild to moderate })}\right)\right]=1 /\left(1+e^{(\text {score-1.01) }}\right)$
$\operatorname{Pr}($ uveitis $<$ severe $)=\left[1 /\left(1+e^{(\text {score-constant moderate to severe })}\right)\right]=1 /\left(1+e^{(\text {score }-4.91)}\right)$ 
Table 1 Description of demographic characteristics and bivariate analysis

\begin{tabular}{|c|c|c|c|c|c|}
\hline \multirow[t]{2}{*}{ Variables } & \multicolumn{5}{|l|}{ Activity levels } \\
\hline & All $(N=195)$ & Mild $(N=77)$ & Moderate $(N=93)$ & Severe $(N=25)$ & $p$ \\
\hline Sex, female, $n(\%)$ & $106(54)$ & $42(55)$ & $52(56)$ & $12(48)$ & 0.770 \\
\hline Age, mean $\left(\mathrm{SD}^{\mathrm{a}}\right)$ & $45.8(16.1)$ & $46.5(15.7)$ & $45.3(17.1)$ & $45.4(13.8)$ & 0.890 \\
\hline Patient $\mathrm{VAS}^{\mathrm{b}}$ median $\left(\mathrm{IQR}^{\mathrm{c}}\right)$ & $5.0(2.0-7.0)$ & $3.0(2.0-6.0)$ & $5.0(2.0-7.0)$ & $7.0(6.0-8.0)$ & $<0.001$ \\
\hline Physician VAS ${ }^{\mathrm{b}}$ median $\left(\mathrm{IQR}^{\mathrm{c}}\right)$ & $4.0(2.0-6.0)$ & $2.0(2.0-3.0)$ & $5.0(3.0-6.0)$ & $7.0(6.0-8.0)$ & $<0.001$ \\
\hline Eye, right $n(\%)$ & $106(54)$ & $40(52)$ & $54(58)$ & $12(48)$ & 0.570 \\
\hline \multicolumn{6}{|l|}{ Anatomic location $n(\%)$} \\
\hline Anterior & $100(51.28)$ & $56(72.73)$ & $37(39.78)$ & $7(28)$ & $<0.001$ \\
\hline Intermediate & $30(15.38)$ & $7(9.09)$ & $23(24.73)$ & $0(0)$ & \\
\hline Posterior & $29(14.87)$ & $3(3.9)$ & $17(18.28)$ & $9(36)$ & \\
\hline Panuveitis & $36(18.46)$ & $11(14.29)$ & $16(17.2)$ & $9(36)$ & \\
\hline Visual acuity median $\left(\mathrm{IQR}^{\mathrm{c}}\right)$ & $0.8(0.4-1.2)$ & $1.0(0.5-1.2)$ & $0.6(0.3-1.0)$ & $0.4(0.2-0.8)$ & $<0.001$ \\
\hline Anterior chamber cell grade $n(\%)$ & & & & & $<0.001$ \\
\hline 0 & $49(25)$ & $9(12)$ & $32(34)$ & $8(32)$ & \\
\hline $1+$ & $59(30)$ & $36(47)$ & $18(19)$ & $5(20)$ & \\
\hline $2+$ & $48(25)$ & $26(34)$ & $21(23)$ & $1(4)$ & \\
\hline $3+$ & $32(16)$ & $6(8)$ & $20(22)$ & $6(24)$ & \\
\hline $4+$ & $7(4)$ & $0(0)$ & $2(2)$ & $5(20)$ & \\
\hline Hypopyon median $\left(\mathrm{IQR}^{\mathrm{c}}\right)$ & $0.0(0.0-0.0)$ & $0.0(0.0-0.0)$ & $0.0(0.0-0.0)$ & $0.0(0.0-0.0)$ & 0.620 \\
\hline Fibrin in the anterior chamber $n(\%)$ & $18(9)$ & $4(5)$ & $10(11)$ & $4(16)$ & 0.197 \\
\hline Keratic precipitates $n(\%)$ & $104(53)$ & $42(55)$ & $48(52)$ & $14(56)$ & 0.890 \\
\hline Iris nodules $n(\%)$ & $6(3)$ & $4(5)$ & $2(2)$ & $0(0)$ & 0.500 \\
\hline Intraocular pressure $n(\%)$ & & & & & 0.013 \\
\hline$\leq 21 \mathrm{mmHg}$ & $178(91)$ & $73(95)$ & $86(92)$ & $19(76)$ & \\
\hline$>21 \mathrm{mmHg}$ & $17(9)$ & $4(5)$ & $7(8)$ & $6(24)$ & \\
\hline Vitreous haze $n(\%)$ & & & & & $<0.001$ \\
\hline Null & $117(60)$ & $59(77)$ & $49(53)$ & $9(36)$ & \\
\hline Mild (1-2+) & $49(25)$ & $15(19)$ & $27(29)$ & $7(28)$ & \\
\hline Severe $(3-4+)$ & $26(13)$ & $3(4)$ & $16(17)$ & $7(28)$ & \\
\hline Presence of snowballs $n(\%)$ & $23(12)$ & $6(8)$ & $15(16)$ & $2(8)$ & 0.220 \\
\hline Snowbanks: number of clock hours, median $\left(\mathrm{IQR}^{\mathrm{c}}\right)$ & $0.0(0.0-0.0)$ & $0.0(0.0-0.0)$ & $0.0(0.0-0.0)$ & $0.0(0.0-0.0)$ & 0.640 \\
\hline Macular edema $n(\%)$ & & & & & $<0.001$ \\
\hline$\leq 315 \mu \mathrm{m}$ & $155(79)$ & $72(94)$ & $63(68)$ & $20(80)$ & \\
\hline$>315 \mu \mathrm{m}$ & $40(21)$ & $5(6)$ & $30(32)$ & $5(20)$ & \\
\hline $\begin{array}{l}\text { Choroidal or retinal lesions: maximal size } \\
\text { (disk diameter) median }\left(\mathrm{IQR}^{\mathrm{c}}\right)\end{array}$ & $0.0(0.0-0.0)$ & $0.0(0.0-0.0)$ & $0.0(0.0-0.0)$ & $0.0(0.0-0.3)$ & 0.198 \\
\hline Number of choroidal or retinal lesions $n(\%)$ & & & & & 0.009 \\
\hline 0 & $166(85)$ & $72(94)$ & $77(83)$ & $17(68)$ & \\
\hline $1-5$ & $16(8)$ & $4(5)$ & $9(10)$ & $3(12)$ & \\
\hline$\geq 6$ & $12(6)$ & $1(1)$ & $7(8)$ & $4(16)$ & \\
\hline Exudative retinal detachment $n(\%)$ & $4(2)$ & $0(0)$ & $4(4)$ & $0(0)$ & 0.185 \\
\hline Inflammatory vessel sheathing $n(\%)$ & $38(19)$ & $7(9.1)$ & $19(20.4)$ & $12(48)$ & $<0.001$ \\
\hline Papillitis $n(\%)$ & $18(9)$ & $2(2.6)$ & $10(10.8)$ & $6(24)$ & 0.004 \\
\hline
\end{tabular}

${ }^{\mathrm{a}} S D$ standard deviation, ${ }^{\mathrm{b}} V A S$ visual analog scale, ${ }^{\mathrm{c}} I Q R$ interquartile interval

included in the study. Demographic characteristics and bivariate analysis are summarized in Table 1 . The mean age was $45.8 \pm 16.1$ years; $54 \%(n=106)$ were female.
Forty-eight percent $(n=93)$ presented moderate activity, $39 \%(n=77)$ mild activity, and $13 \%(n=25)$ severe activity. 
Table 2 Multivariate model of uveitis activity level in the study population

\begin{tabular}{lcc}
\hline Variables & $\mathrm{OR}_{\text {adjusted }}(95 \% \mathrm{CI})$ & $\mathrm{LOG}\left(\mathrm{OR}^{\mathrm{a}}\right)$ \\
\hline Anterior chamber cell grade (ref. 0) & \\
$1+$ & $0.24(0.10-0.60)$ & -1.41 \\
$2+$ & $0.49(0.19-1.27)$ & -0.72 \\
$3+$ & $2.97(1.04-8.49)$ & 1.09 \\
$4+$ & $27.85(3.42-226.75)$ & 3.33 \\
Vitreous haze (ref. Null) & $1.46(0.65-3.27)$ & 0.38 \\
Mild (1-2+) & $3.95(1.40-11.16)$ & 1.37 \\
Severe (3-4+) & $3.58(1.56-8.21)$ & 1.28 \\
Macular edema, $\mu \mathrm{m}$ & & \\
$\quad$ ref. $\leq 315 \mu \mathrm{m})$ & $4.43(1.71-11.53)$ & 1.49 \\
Inflammatory vessel sheathing & & \\
$\quad$ ref. No) & $1.23(1.09-1.39)$ & 0.21 \\
Patient evaluation & $4.05(1.18-13.92)$ & 1.40 \\
Papillitis (ref. No) & & \\
Number of choroidal or retinal lesions (ref. 0) & \\
1-5 & $2.00(0.63-6.35)$ & 0.69 \\
$\geq 6$ & $4.99(1.08-23.01)$ & 1.61 \\
Constant & & \\
Mild to moderate & & 1.01 \\
Moderate to severe & & \\
\hline
\end{tabular}

${ }^{\text {a }} \operatorname{LOG}(\mathrm{OR})$ logistic regression (odds ratio)

The results of the regression model summarized in Table 2 revealed that the uveitis activity levels were more severe in patients with high degrees of anterior chamber cell grade, high degrees of vitreous haze, central macular edema over $315 \mu \mathrm{m}$, who presented inflammatory vessel sheathing, papillitis, an elevated number of choroidal/retinal lesions and higher patient evaluation.

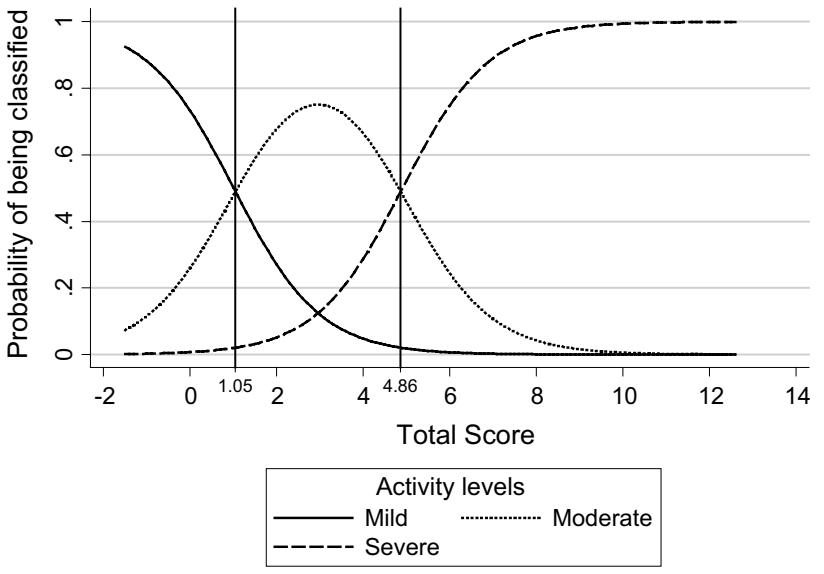

Fig. 1 Probability of being classified with mild, moderate or severe uveitis according to the total score obtained

in Fig. 1. We plotted all of the possible values for the total score along the $\mathrm{X}$-axis and the probability associated with each score along the Y-axis. The three curves represent the fluctuation in probability and the cross-point between curves that allowed for the determination of a score threshold from which a uveitis status could be assigned (see Supplementary Material for an example of calculations). The range of possible values for the scores was approximately $-1 \times 41$ to $12 \times 580$. As can be observed in the graph, patients with a total score below $1 \times 05$ will be classified in the category of mild uveitis; those with a total score between 1.06 and 4.86 will be classified as having moderate uveitis; and those with a total score greater or equal to $4 \times 87$ will be classified as having severe uveitis.

Therefore, clinicians using this tool will only need to substitute the values for AC cell grade, vitreous haze,

$$
\begin{aligned}
\text { UVEDAI } & =\left\{\begin{array}{c}
0.00 \text { if } \mathrm{ACC}=0 \\
-1.41 \text { if } \mathrm{ACC}=1 \\
-0.72 \text { if } \mathrm{ACC}=2 \\
1.09 \text { if } \mathrm{ACC}=3 \\
3.33 \text { if } \mathrm{ACC}=4
\end{array}\right\}+\left\{\begin{array}{c}
0.00 \text { if } \mathrm{VH}=\text { null } \\
0.38 \text { if } \mathrm{VH}=\text { mild } \\
1.37 \text { if } \mathrm{VH}=\text { severe }
\end{array}\right\}+\left\{\begin{array}{l}
0.00 \text { if } \mathrm{ME} \leq 315 \\
1.28 \text { if } \mathrm{ME}>315
\end{array}\right\} \\
& +\left\{\begin{array}{l}
0.00 \text { if } \mathrm{VS}=\text { no } \\
1.49 \text { if } \mathrm{VS}=\text { yes }
\end{array}\right\}+0.21 x \mathrm{PE}+\left\{\begin{array}{l}
0.00 \text { if papilitis }=\text { no } \\
1.40 \text { if papilitis }=\text { yes }
\end{array}\right\} \\
& +\left\{\begin{array}{l}
0.00 \text { if no. } \mathrm{RL}=0 \\
0.69 \text { if no. } \mathrm{RL}=1 \text { to } 5 \\
1.61 \quad \text { if no. } \mathrm{RL} \geq 6
\end{array}\right\}
\end{aligned}
$$

ACC: anterior chamber cell grade; VH: vitreous haze; VS: inflammatory vessel sheathing; PE: patient evaluation; RE: choroidal/retinal lesions.

The distribution of the probabilities of being classified in each of the 3 uveitis categories-based on the score obtained and the cut points for each category-is shown macular edema, inflammatory vessel sheathing, patient evaluation, papillitis and the number of choroidal/retinal lesions in the formula given, and with the score obtained, to classify a patient's uveitis disease activity level according to the established cut points: $\leq 1 \times 05$ : mild; $1 \times 06$ to $\leq 4 \times 86$ : moderate; $\geq 4 \times 87$ : severe. 
(a)

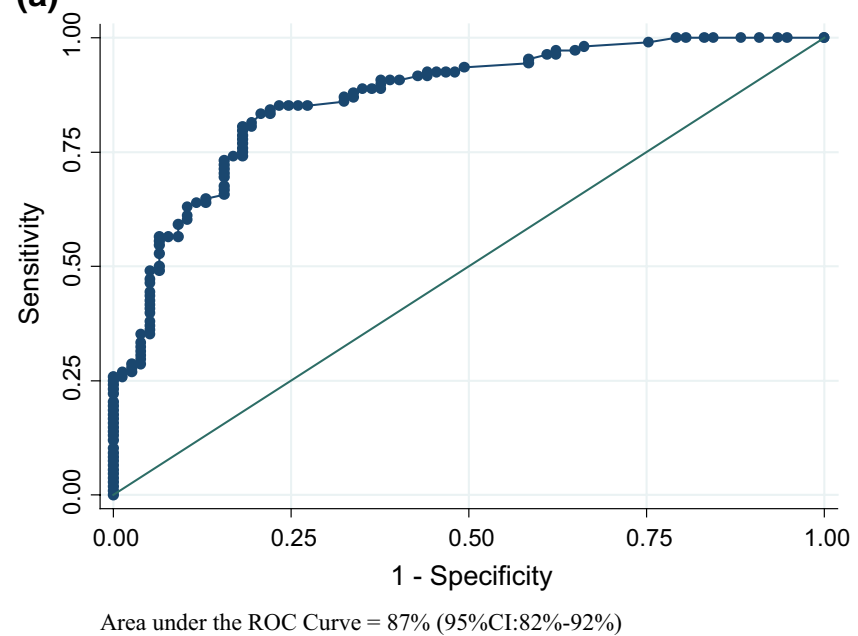

(b)

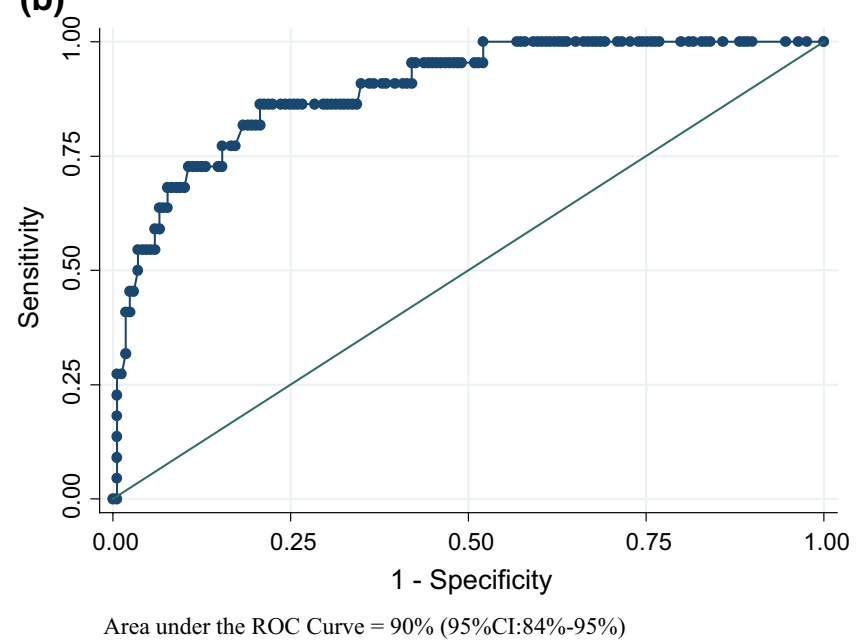

Fig. 2 Receiver operating characteristic (ROC) curve and AUC for the predictive modeling accuracy to discriminate. a Patients with mild UVEITIS from patients with moderate or severe UVEITIS, b patients with mild or moderate UVEITIS from patients with severe UVEITIS

The discriminatory capacity of the resulting multivariate model was $87 \%$ (95\% CI $82-92 \%$ ) for differentiating patients with mild uveitis from those with moderate or severe uveitis and 90\% (95\% CI 84-95\%) for differentiating patients with mild or moderate uveitis from those with severe uveitis (Fig. 2). Additionally the results from the analysis for the internal validation revealed that for none of the 1000 replicates of the random distribution of the outcome the resulting AUC was better than the original, meaning the probability of obtaining by chance an AUC better than the obtained with the original model is $<0.001$.

\section{Discussion}

In our knowledge, this is the first study in which it is developed an activity index (UVEDAI) that attempts to classify patients with uveitis according to the degree of their ocular inflammatory activity. The results reported herein could be of interest to ophthalmologists, rheumatologists and other medical specialties that keep abreast of clinical investigation into systemic diseases with ocular involvement. Of all the variables investigated, anterior chamber cell grade, vitreous haze, central macular edema, inflammatory vessel sheathing, papillitis, choroidal/retinal lesions and patient evaluation were the most important to determining the activity of uveitis. Our score showed an $87 \%$ capacity to differentiate patients with mild uveitis from those with moderate or severe uveitis and an $88 \%$ capacity for differentiating patients with mild or moderate uveitis from those with severe uveitis.

Uveitis occurs in many patients who have a systemic rheumatic disease. In those with an established diagnosis of autoimmune disease, ocular inflammation can mark the severity of the systemic condition. We believe that assessment of ocular activity as an index can be useful in the global management of patients with systemic inflammatory diseases, especially in the assessment of treatment response.

The evaluation of cells in the anterior chamber is the accepted standard procedure for assessing inflammatory activity in the anterior segment $[4,25]$. It is worth remarking the protective effect of categories $1\left(\mathrm{OR}_{\text {adjusted }}\right.$ $0.24 ; 95 \%$ CI $0.10-0.60)$ and $2\left(\mathrm{OR}_{\text {adjusted }} 0.49 ; 95 \% \mathrm{CI}\right.$ $0.19-1.27)$ of this variable in the multivariate model. The explanation of this effect is that $91.84 \%$ of the patients with grade 0 have intermediate uveitis, posterior or panuveitis not anterior uveitis. In these patients, the overall value of the UVEDAI is not affected because it is corrected from the coefficients of other variables that are involved, such as macular edema, vasculitis or others. Vitreous haze is the most widely accepted measure of inflammation in the vitreous cavity. This variable has often been used as a primary outcome in many randomized clinical trials [26-29]. Other significant factor on said score was macular edema, the most common cause of irreversible visual impairment in patients with uveitis but a treatable entity [30-33]. We believe that its clinical relevance, its relationship with chronic inflammatory activity and the ease of its implementation in an objective and reproducible way with SDOCT confirm the importance of its inclusion in UVEDAI. In the posterior pole, the presence of inflammatory vessel sheathing, choroidal/retinal lesions and papillitis has been established and characterized clinical variables indicating inflammatory activity and their presence is often a warning sign. Although in our study the number of patients with 
these inflammatory events was reduced, they were nonetheless significant. According to this finding, and in line with the new clinical trials, which has incorporated the abovementioned parameters for the routine evaluation of clinical activity in uveitis [29], we regard these variables as truly significant in the composite index that has been developed. Another item bearing an important association is the patient evaluation.

On the other hand, variables, such as visual acuity, intraocular pressure, hypopyon, which are clinically relevant in the routine assessment of patient with uveitis, have not proven to be statistically significant in our study. In the same way, however, they are clinical signs that must be routinely assessed in the evaluation of any eye with uveitis.

The inclusion of the SD-OCT in the assessment of macular edema derives from its wide availability in ophthalmologist outpatient clinics, its noninvasive approach, and the fact that it is considered the gold standard in diagnosing and monitoring of $\mathrm{ME}[31,34]$.

The main strength of the developed UVEDAI is its usefulness as a standardized tool that relies on variables extracted from examinations established in routine clinical practice, without complex equipment-based diagnoses. We believe that this composite index, which assesses global ocular inflammatory activity and classification, will not only allow clinicians to compare the uveitis activity, regardless of cause, but will also serve as a useful tool for future studies and trials when sensitivity to change will be analyzed. Just as in other disease activity score [11], UVEDAI assigns different weightings to each variable based on any of the ocular anatomic regions. Since these are often affected simultaneously, the result is a score that indicates global ocular inflammatory activity, which can accurately be classified as mild, moderate or severe.

One limitation is the use of the ophthalmologist's clinical judgment as the gold standard for classifying inflammatory uveitis activity in mild, moderate and severe cases of the disease. Our decision was based on the fact that no gold standard set of criteria for ocular inflammatory activity with which to compare our index currently exists in the scientific literature. In fact, there are some articles in the literature that define the outcome as an "improvement" in activity according to clinical criteria [35-37]. Furthermore, clinical judgment of the physician was also used in the development, of other composite indices as popular as disease activity score (DAS) for rheumatoid arthritis [11]. Another limitation concerning the development phase of the index is the presence of a possible circularity between the predictor variables and the main variable. We will determine whether this potential circularity affects UVEDAI during an already programed validation phase in which patients will be independently evaluated and in a masked form by two ophthalmologists.
Another limitation that had to be overcome involved measuring the model's discrimination capacity to verify the accuracy of ordinal logistic regression models against existing methods. It must be borne in mind, however, that ordinal logistic regression works under the assumption of proportional odds across responses; that is, each explanatory variable has the same effect at each cumulative split of the ordinal dependent variable (effect of mild vs. moderate + severe $\approx$ effect of mild + moderate vs. severe). Keeping this in mind, and taking into account that our data did satisfy the proportional odds assumption, the use of two AUCs to measure the capacity to differentiate between patients with mild from those with moderate or severe uveitis, as well as between patients with mild or moderate from those with severe uveitis, gives a good approximation of the total model accuracy.

In conclusion, the UVEDAI is the first ocular easy-to-use composite score based on variables commonly used in clinical practice for uveitis assessment that assesses and classifies global ocular inflammatory activity with high discriminatory power. We believe that it could be a useful tool not only in daily clinical practice, but also for comparing results in clinical and therapeutic studies. Further studies are required to validate this index and analyze its metric capacity.

Acknowledgements This study was conducted with an unrestricted grant from Abbvie. We would also like to thank the following individuals who directly contributed to this study: Professor Alfredo Adán Civera, MD PhD; Professor Javier Llorca Díaz, MD PhD; Estibaliz Loza Santamaria, Maria Jesús García Yébenes, MD PhD; Silvia Herrera and Juan Manuel Barrio.

Funding The Spanish Society of Rheumatology is the sponsor and funder of this study and has participated in the study design; in the analysis, and interpretation of data; in the writing of the report; and in the decision to submit the paper for publication. The corresponding author had full access to all study data and had final responsibility for the decision to submit the manuscript for publication.

\section{Compliance with ethical standards}

Conflict of interest All authors declare that no conflicts of interest exist.

Ethical approval This article contains a study with human participants performed by any of the authors. This study was performed following the principles outlined in the Helsinki Declaration, and the study protocol was approved by the ethics committee at all participating hospitals.

Open Access This article is distributed under the terms of the Creative Commons Attribution 4.0 International License (http://creativecommons.org/licenses/by/4.0/), which permits unrestricted use, distribution, and reproduction in any medium, provided you give appropriate credit to the original author(s) and the source, provide a link to the Creative Commons license, and indicate if changes were made. 


\section{References}

1. Yeh S, Faia LJ, Nussenblatt RB (2008) Advances in the diagnosis and immunotherapy for ocular inflammatory disease. Semin Immunopathol 30:145-164. doi:10.1007/s00281-008-0109-4

2. Rothova A, Suttorp-van Schulten MS, Frits Treffers W et al (1996) Causes and frequency of blindness in patients with intraocular inflammatory disease. Br J Ophthalmol 80:332-336

3. Bodaghi B, Cassoux N, Wechsler B et al (2001) Chronic severe uveitis: etiology and visual outcome in 927 patients from a single center. Medicine (Baltimore) 80:263-270

4. Jabs DA, Nussenblatt RB, Rosenbaum JT et al (2005) Standardization of uveitis nomenclature for reporting clinical data. Results of the first international workshop. Am J Ophthalmol 140:509-516

5. Trusko B, Thorne J, Jabs D et al (2013) The Standardization of Uveitis Nomenclature (SUN) Project. Development of a clinical evidence base utilizing informatics tools and techniques. Methods Inf Med 52:259-265. doi:10.3414/ME12-01-0063

6. Bloch-Michel E, Nussenblatt RB (1987) International Uveitis Study Group recommendations for the evaluation of intraocular inflammatory disease. Am J Ophthalmol 103:234-235

7. de Smet MD, Taylor SR, Bodaghi B et al (2011) Understanding uveitis: the impact of research on visual outcomes. Prog Retin Eye Res 30:452-470. doi:10.1016/j.preteyeres.2011

8. Pato E, Munoz-Fernandez S, Francisco F et al (2011) Systematic review on the effectiveness of immunosuppressants and biological therapies in the treatment of autoimmune posterior uveitis. Semin Arthritis Rheum 40:314-323. doi:10.1016/j. semarthrit.2010.05.008

9. Denniston AK, Holland GN, Kidess A et al (2015) Heterogeneity of primary outcome measures used in clinical trials of treatments for intermediate, posterior, and panuveitis. Orphanet J Rare Dis 10:97. doi:10.1186/s13023-015-0318-6

10. van der Heijde DM, van't Hof MA, van Riel PL et al (1990) Judging disease activity in clinical practice in rheumatoid arthritis: first step in the development of a disease activity score. Ann Rheum Dis 49:916-920

11. van der Heijde DM, van 't Hof MA, van Riel PL et al (1993) Development of a disease activity score based on judgment in clinical practice by rheumatologists. J Rheumatol 20:579-581

12. Lukas C, Landewe R, Sieper J et al (2009) Development of an ASAS-endorsed disease activity score (ASDAS) in patients with ankylosing spondylitis. Ann Rheum Dis 68:18-24. doi:10.1136/ ard.2008.094870

13. Tugwell P, Boers M, Brooks $P$ et al (2007) OMERACT: an international initiative to improve outcome measurement in rheumatology. Trials 8:38

14. Escobar-Pérez J, Cuervo-Martínez A (2008) Validez de contenido y juicio de expertos: una aproximación a su utilización. http:// www.humanas.unal.edu.co/psicometria/files/7113/8574/5708/ Articulo3_Juicio_de_expertos_27-36.pdf. Accessed 20 June 2016

15. Heinz C, Schumacher C, Roesel M et al (2012) Elevated intraocular pressure in uveitis associated with juvenile idiopathic arthritis-associated uveitis, often detected after achieving inactivity. $\mathrm{Br} \mathrm{J}$ Ophthalmol 96:140-141. doi:10.1136/ bjophthalmol-2011-300731

16. Whitcup SM (2004) Examination of the patient with uveitis. In: Mosby (ed) Uveitis fundamentals and clinical practice. Elsevier, St Louis, pp 54-65

17. Nussenblatt RB, Palestine AG, Chan CC et al (1985) Standardization of vitreal inflammatory activity in intermediate and posterior uveitis. Ophthalmology 92:467-471
18. Grover S, Murthy RK, Brar VS et al (2009) Normative data for macular thickness by high-definition spectral-domain optical coherence tomography (spectralis). Am J Ophthalmol 148:266271. doi:10.1016/j.ajo.2009.03.006

19. Peduzzi P, Concato J, Feinstein AR et al (1995) Importance of events per independent variable in proportional hazards regression analysis. II. Accuracy and precision of regression estimates. J Clin Epidemiol 48:1503-1510

20. Concato J, Peduzzi P, Holford TR et al (1995) Importance of events per independent variable in proportional hazards analysis. I. Background, goals, and general strategy. J Clin Epidemiol 48:1495-1501

21. Peduzzi P, Concato J, Kemper E et al (1996) A simulation study of the number of events per variable in logistic regression analysis. J Clin Epidemiol 49:1373-1379

22. Hajian-Tilaki K (2013) Receiver operating characteristic (ROC) curve analysis for medical diagnostic test evaluation. Casp $\mathbf{J}$ Intern Med 4:627-635

23. Hanley JA (1989) Receiver operating characteristic (ROC) methodology: the state of the art. Crit Rev Diagn Imaging 29:307-335

24. Linnet K (1987) Comparison of quantitative diagnostic tests: type I error, power, and sample size. Stat Med 6:147-158

25. Heiligenhaus A, Foeldvari I, Edelsten C et al (2012) Proposed outcome measures for prospective clinical trials in juvenile idiopathic arthritis-associated uveitis: a consensus effort from the multinational interdisciplinary working group for uveitis in childhood. Arthritis Care Res (Hoboken) 64:1365-1372. doi:10.1002/acr.2167

26. Dick AD, Tugal-Tutkun I, Foster S et al (2013) Secukinumab in the treatment of noninfectious uveitis: results of three randomized, controlled clinical trials. Ophthalmology 120:777-787. doi:10.1016/j.ophtha.2012.09.040

27. Lowder C, Belfort R Jr, Lightman S et al (2011) Dexamethasone intravitreal implant for noninfectious intermediate or posterior uveitis. Arch Ophthalmol 129:545-553. doi:10.1001/ archophthalmol.2010.339

28. Letko E, Yeh S, Foster CS et al (2015) Efficacy and safety of intravenous secukinumab in noninfectious uveitis requiring steroid-sparing immunosuppressive therapy. Ophthalmology 122:939-948. doi:10.1016/j.ophtha.2014.12.033

29. Jaffe GJ, Dick AD, Brézin AP, Nguyen QD, Thorne JE et al (2016) Adalimumab in patients with active noninfectious uveitis. N Engl J Med 375:932-943. doi:10.1056/NEJMoa1509852

30. Lardenoye CW, van Kooij B, Rothova A (2006) Impact of macular edema on visual acuity in uveitis. Ophthalmology 113:1446-1449

31. Iannetti L, Spinucci G, Abbouda A et al (2012) Spectral-domain optical coherence tomography in uveitic macular edema: morphological features and prognostic factors. Ophthalmologica 228:13-18. doi:10.1159/000337234

32. Karampelas M, Sim DA, Chu C et al (2015) Quantitative analysis of peripheral vasculitis, ischemia, and vascular leakage in uveitis using ultra-widefield fluorescein angiography. Am J Ophthalmol 159:1161-1168. doi:10.1016/j.ajo.2015.02.009

33. Hunter RS, Skondra D, Papaliodis G et al (2012) Role of OCT in the diagnosis and management of macular edema from uveitis. Semin Ophthalmol 27:236-241. doi:10.3109/08820538.2012.70 8813

34. Sugar EA, Jabs DA, Altaweel MM et al (2011) Identifying a clinically meaningful threshold for change in uveitic macular edema evaluated by optical coherence tomography. Am J Ophthalmol 152:1044-1052. doi:10.1016/j.ajo.2011.05.028

35. Pacor ML, Biasi D, Lunardi C et al (1994) Cyclosporin in Behcet's disease: results in 16 patients after 24 months of therapy. Clin Rheumatol 13:224-227 
36. Miserocchi E, Baltatzis S, Ekong A et al (2002) Efficacy and safety of chlorambucil in intractable noninfectious uveitis: the Massachusetts Eye and Ear Infirmary experience. Ophthalmology 109:137-142
37. Vianna RN, Ozdal PC, Deschenes J et al (2006) Combination of azathioprine and corticosteroids in the treatment of serpiginous choroiditis. Can J Ophthalmol 41:183-189 